\title{
Interactions within Distributed Mixed Reality Collaborative Environments
}

\author{
Anasol Peña-Ríos *, Vic Callaghan *, Michael Gardner *, Mohammed J. Alhaddad * \\ * Department of Computer Science, University of Essex, UK. E-mail: acpena@essex.ac.uk \\ ${ }^{\dagger}$ Faculty of Computing and Information Technology, King Abdulaziz University, KSA.
}

\section{INTRODUCTION}

Traditionally virtual worlds have been regarded as standalone entities. However, the world moves fast towards a mixed reality collective environment, joining virtual and real world by incorporating accessible ubiquitous computing for people. Mobile and wearable computers act as a door to connect people to virtuality, e.g. the use of fitness/activity trackers, which collect real world information helping users to complement reality with virtuality improving their health and fitness. A different example is the use of mobile devices to connect people that do not share the same physical location in a virtual way, thought phone calls, videoconferences, chat and social media applications. These examples show that currently we live in two realities, processing information of both worlds in real time.

Our video submission presents a work-in-progress research prototype towards the creation of a Blended Reality Distributed System, complementing the paper [1] submitted to the main track of the conference. The test bed scenario proposed is a mixed reality collaborative laboratory activity, performed by learners within geographically dispersed locations. The goal of the activity is to construct a small robot emphasising computing fundamentals. The video is available at: http://youtu.be/akKPHnDY9bw .

\section{A BLENDED REALity Distributed System}

Blended reality can be defined as the interaction performed by a user within two different environments (real/local and virtual/distant); extending them to work as if they were one in real-time, by unconsciously merging traces of one into the other; and often being perceived as simultaneously [2]. Currently, this action is performed by the people, in this increasingly mixed reality world; e.g. in virtual meetings where people in distant locations can be connected through a virtual space, in order to achieve communication and collaboration. Some researchers propose the use of tangible user interfaces (TUI) in order to augment "the real physical world by coupling digital information to everyday physical objects and environments" [3].

Fig. 1 shows possible interactions between users and TUIs within mixed reality environments. A number of implementations are based on unidirectional communication, in which users can collaborate in a virtual space, but the action can only be followed and shared in the virtual world. In [4], we proposed the use of xReality objects to achieve collaborative bi-directional communications between real and virtual objects; allowing sharing both, real and virtual objects. $x$ Reality objects are "smart networked objects coupled to their virtual representation, updated and maintained in real time to create a mirrored state (dual reality)" [5].

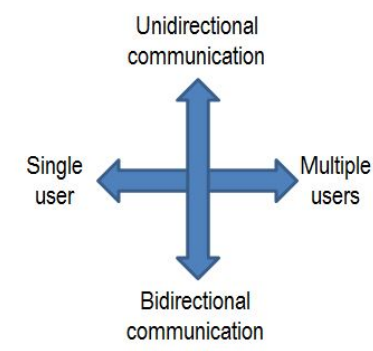

Figure 1. Interactions between users and a mixed reality environment.

\section{IMPLEMENTATION}

Fig. 2 describes the architecture proposed to create and share dual reality between multiple users. To implement the real/physical side of a xReality object we utilise two components: 1) a collection of pluggable hardware boards for rapid prototyping, which can be interconnected in diverse combinations to create a variety of quick Internetof-Things (IoT) prototypes (Fortito's BuzzBoard Educational Toolkit ${ }^{1}$ ) and 2) a Raspberry $\mathrm{Pi}^{2}$, a low cost small computer used in electronic hobbyist projects and education. The virtual representation was developed using Unity $3 \mathrm{D}^{3}$, a cross-platform game engine used for videogames and $3 \mathrm{D}$ content. To mirror and synchronize virtual representations we use SmartFoxServer X2 ${ }^{4}$ (SFS2X), a middleware to create large scale multiplayer games and virtual communities.

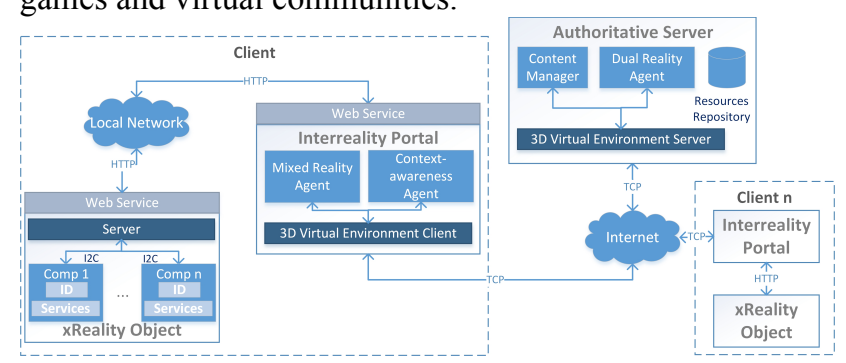

Figure 2. Blended Reality Distributed System architecture [6].

\section{CLASSIFICATION OF INTERACTIONS}

In previous works [5] we defined six possible scenarios for interaction between real objects, virtual objects and

\footnotetext{
${ }^{1}$ Fortito Ltd - http://www.fortito.com/en

2 Raspberry Pi Foundation - http://www.raspberrypi.org

${ }^{3}$ Unity3D Game Engine - www.unity3d.com

${ }^{4}$ SmartFoxServer $2 \mathrm{X}$ - www.smartfoxserver.com
} 
users inside a common blended reality environment (Fig. 2). We classified these scenarios in base of the number of users interacting within a session and the relationships between real and virtual objects:

\section{1) Individual session}

a) Scenario 1 (S1) describes the use of virtual objects only. As there is no real object involved, none of the actions performed in the virtual world is mirrored to a real counterpart, therefore this scenario describes the use of simulation only $(0: 1)$.

b) Scenario $2(\mathrm{~S} 2)$ represents the ideal single dualreality-state; in which, one real object is mirrored in real time to one virtual object $(1: 1)$.

2) Collaborative session

a) Scenario 3 (S3) examines the use of a virtual object shared by multiple users. Similarly to S1, as the users do not interact with a real object, the scenario is based on simulation $(0: 1)$.

b) Scenario 4 (S4) defines a partial dual-reality-state; in which, one real object is mirrored in real time to one virtual object $(1: 1)$. In this case both users can interact with the xReality object via its virtual representation but only one of them will see the mirrored action in the real world. If an action is triggered in the real object by the local user, then both, the remote and the local user can perceive the reaction reflected in the virtual world.

c) Scenario 5 (S5) explains the ideal multiple dualreality-state, where all real objects are connected to the same virtual representation (n:1). In this case any action executed in the real or the virtual world can be mirrored to their counterparts and extended to all the users within the session.

d) Scenario 6 (S6) outlines a complementary dualreality-state; in which, each one of the users has a piece of the puzzle to construct an object. Imagine that two users are creating a robot in collaboration, user $\mathrm{A}$ has the motion component (e.g. servo motor) and user B has a light follower sensor which will be used to move the robot. They can construct a robot in the virtual world able to move in the direction sent by user B's component, whilst user A can observe how his component matches the movement of the virtual robot (n:n).

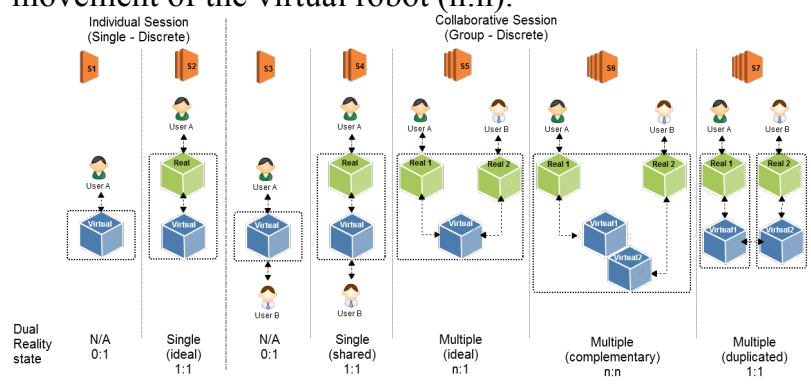

Figure 3. Interactions within a Blended Reality environment [5]. e) Scenario 7 (S7) shows a duplicated ideal single dual-reality-state, where real objects reflect their virtual counterparts (1:1). However, in this case we have two xReality objects instead of one; and they can communicate through their virtual components.

\section{SUMMARY AND FUTURE WORK}

Our video submission presents a work-in-progress research towards the implementation of the interactions between users and xReality objects defined in the previous section. The video shows execution of single dual-realitystates (ideal and shared) in individual and collaborative sessions, as described in scenarios 1-4. This opens opportunities for collaboration and development in education, commercial R\&D, medicine or even hackerspaces and hobbyists activities.

Future work plans include the implementation of multiple dual realities (ideal, complementary and duplicated) using two or more xReality objects; and the integration of sequenced groups of services to be executed within our mixed reality environment.

\section{ACKNOWLEDGMENT}

We are pleased to acknowledge King Abdulaziz University, Saudi Arabia for their generous funding of this research project, including the provision of a $\mathrm{PhD}$ scholarship to the lead author. In addition, we wish to thank Immersive Displays UK Ltd. and Fortito Ltd. for their support.

\section{REFERENCES}

[1] A. Pena-Rios, V. Callaghan, M. Gardner and M. Alhaddad, "Using mixed-reality to develop smart environments" submitted to the IEEE 10th International Conference on Intelligent Environments 2014 (IE'14), Shanghai, China, 2014.

[2] S. A. Applin and M. Fischer, "A Cultural Perspective on Mixed , Dual and Blended Reality," in IUI - Workshop on Location Awareness for Mixed and Dual Reality (LAMDa'11), Palo Alto California, USA, 2011.

[3] H. Ishii and B. Ulmer, "Tangible bits: towards seamless interfaces between people, bits, and atoms," in Conference on Human Factors in Computing Systems (CHI'97), Atlanta, 1997.

[4] A. Peña-Rios, V. Callaghan, M. Gardner and M. Alhaddad, "Remote mixed reality collaborative laboratory activities: Learning activities within the InterReality Portal," in The Intelligent Campus International Symposium (IC'12), Macau, 2012.

[5] A. Pena-Rios, V. Callaghan, M. Gardner and M. J. Alhaddad, "xReality interactions within a mixed reality learning environment," in 3rd European Immersive Education Summit, London, 2013.

[6] A. Peña-Rios, V. Callaghan, M. Gardner and M. J. Alhaddad, "Developing xReality objects for mixed - reality environments," in Workshop Proceedings of the 9th International Conference on Intelligent Environments, Athens, Greece, 2013. 